\title{
Structure, Function, and Regulation of the Essential Virulence Factor Capsular Polysaccharide of Vibrio vulnificus
}

\author{
Gregg S. Pettis *(D) and Aheli S. Mukerji \\ Department of Biological Sciences, Louisiana State University, Baton Rouge, LA 70803, USA; amuker1@lsu.edu \\ * Correspondence: gpettis@lsu.edu; Tel.: +1-225-578-2798
}

Received: 29 February 2020; Accepted: 3 May 2020; Published: 5 May 2020

\begin{abstract}
Vibrio vulnificus populates coastal waters around the world, where it exists freely or becomes concentrated in filter feeding mollusks. It also causes rapid and life-threatening sepsis and wound infections in humans. Of its many virulence factors, it is the V. vulnificus capsule, composed of capsular polysaccharide (CPS), that plays a critical role in evasion of the host innate immune system by conferring antiphagocytic ability and resistance to complement-mediated killing. CPS may also provoke a portion of the host inflammatory cytokine response to this bacterium. CPS production is biochemically and genetically diverse among strains of $V$. vulnificus, and the carbohydrate diversity of CPS is likely affected by horizontal gene transfer events that result in new combinations of biosynthetic genes. Phase variation between virulent encapsulated opaque colonial variants and attenuated translucent colonial variants, which have little or no CPS, is a common phenotype among strains of this species. One mechanism for generating acapsular variants likely involves homologous recombination between repeat sequences flanking the $w z b$ phosphatase gene within the Group 1 CPS biosynthetic and transport operon. A considerable number of environmental, genetic, and regulatory factors have now been identified that affect CPS gene expression and CPS production in this pathogen.
\end{abstract}

Keywords: Vibrio; capsular polysaccharide; phase variation; sepsis; wound infection

\section{Introduction}

Vibrio vulnificus is a natural inhabitant of estuarine and other coastal marine environments where it exists free living or becomes concentrated in filter-feeding molluscan shellfish, such as oysters. The bacterium gains entrance to the human host through consumption of contaminated water or food, particularly raw or undercooked oysters and other seafood. Alternatively, infection occurs through open wounds that contact seawater or shellfish containing the bacterium [1]. V. vulnificus notoriously causes an acute fulminating septicemia as well as severe wound infections. A third illness, gastroenteritis, is not life threatening and is typically not reported. Septicemic patients succumb to death in as little as 24 hours and in over $50 \%$ of cases. V. vulnificus is responsible for the vast majority of reported deaths associated with seafood consumption in the U.S. [1]. Patients developing sepsis through oral ingestion of the bacterium overwhelmingly have an underlying predisposing health condition such as liver disease, AIDS, diabetes, hemachromatosis, cancer, or a compromised immune system [2]. Infection of wounds occurs typically during swimming, fishing, or handling of seafood. Such infections can progress rapidly to a necrotizing fasciitis at the site of infection, and they often require debridement or amputation of the affected limb in order to prevent death, which occurs in $\sim 25 \%$ of cases $[3,4]$.

A number of factors contribute to virulence of $V$. vulnificus, including its acid resistance, as well as its production of capsular polysaccharide (CPS), lipopolysaccharide (LPS), iron acquisition systems, 
cytotoxic factors, and motility and adherence/adhesion molecules [3]. Unlike most of the other contributing factors, however, CPS is considered to be essential for virulence, since it confers the ability to circumvent the host immune response [3,5]. Here, we summarized research aimed at elucidating $V$. vulnificus CPS structure, function, and genetics, as well as control of CPS production via phase variation and other known environmental, genetic, and regulatory factors.

\section{Identification of the V. vulnificus Capsule and Its Correlation with Virulence}

Kreger et al. (1981) first presented evidence that virulence of $V$. vulnificus was due, at least in part, to an antiphagocytic surface molecule [6]. In a follow-up study, physicochemical analysis of the partially purified molecule suggested that it was a heat-sensitive acidic polysaccharide [7]. Ruthenium red staining, coupled with transmission electron microscopy, provided evidence that this polysaccharide densely coated the cell surface of a virulent strain while it was present in reduced amounts on the surface of a weakly virulent one [7]. Amako et al. (1984) showed the ruthenium red staining patterns of two V. vulnificus strains, namely FCC and ATCC 27562 (Table 1), were distinct: while both strains showed a mixed population of stained and unstained cells, the proportion of stained cells was greater for strain FCC than ATCC 27562. Moreover, the increased proportion of stained cells correlated with increased virulence in mice and to resistance to the bacteriocidal action of normal human serum (NHS). These authors referred to this surface polysaccharide as a capsule [8].

Table 1. V. vulnificus strains discussed in this review.

\begin{tabular}{|c|c|c|c|}
\hline Strain & Source & CPS Phenotype & CPS Group \\
\hline ATCC $27562^{1}$ & Clinical & Opaque & 4 \\
\hline FCC & Clinical & Opaque $^{2}$ & N.D. ${ }^{3}$ \\
\hline $\mathrm{MO} 6-24 / \mathrm{O}$ & Clinical & Opaque & 1 \\
\hline MO6-24/T & Phase variant of MO6-24/O & Translucent & 1 \\
\hline BO62316 & Clinical & Opaque & N.D. \\
\hline C7184 & Clinical & Opaque & N.D. \\
\hline $1003(\mathrm{O})$ & Clinical & Opaque & N.D. \\
\hline
\end{tabular}

Separately, variation in capsule production among four other $V$. vulnificus strains was similarly characterized [9]. Opaque colony types of strains consisted of encapsulated cells while the cells of translucent colony types produced little or no observable capsule (see Figure 1 for examples of opaque and translucent colony types of $V$. vulnificus). The presence of capsular material again correlated with increased virulence in mice and with resistance to NHS, as well as with antiphagocytic ability, and increased invasiveness in subcutaneous tissue of guinea pigs [9]. In a more comprehensive study, Simpson et al. (1987) examined a larger number of both clinical and environmental isolates for colony phenotype, virulence in mice, and other properties. Twenty-six virulent strains produced both opaque and translucent colony types, while ten strains displayed only the translucent colony type and were avirulent [10]. Thus, a correlation between CPS and virulence in V. vulnificus was apparent.

This relationship was subsequently explored further using transposon mutagenesis. Compared to the opaque clinical isolate MO6-24/O (Table 1), two translucent transposon mutant derivatives appeared acapsular in carbohydrate staining procedures and were attenuated by several orders of magnitude for virulence in mice [11]. Moreover, while only opaque colony-yielding bacteria were recovered post infection from surviving mice infected with MO6-24/O, only bacteria of a translucent colony type (or no bacteria at all) were recovered from mice infected with the acapsular transposon mutants. A spontaneous translucent variant of MO6-24/O, strain MO6-24/T (Table 1), which showed reduced CPS on its cell surface, was also attenuated for virulence though not to the same magnitude as the acapsular transposon mutants. Bacteria recovered from mice infected with MO6-24/T all had an opaque 
morphology, indicating that phase switching from translucent back to opaque had likely occurred in vivo by an undetermined mechanism [11]. Attenuation of virulence was a common property seen for acapsular transposon mutants of V. vulnificus in other studies as well [12-14].

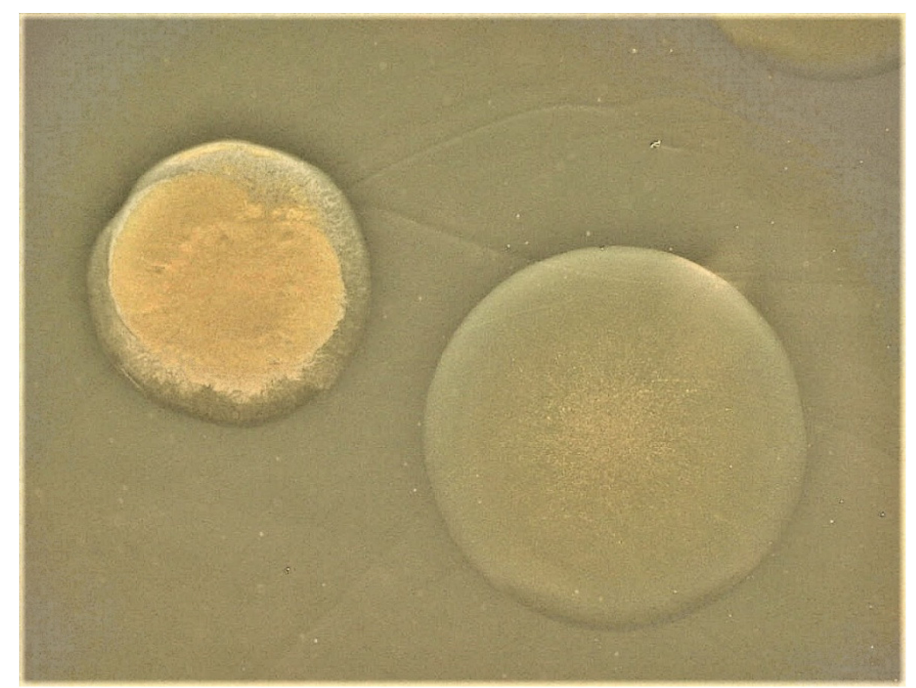

Figure 1. Opaque (left) and translucent (right) colony types of V. vulnificus.

\section{CPS Composition and Structure}

Carbohydrate composition of $V$. vulnificus CPS was first reported for the clinical isolate MO6-24/O following extensive purification of its capsular material and analysis by nuclear magnetic resonance (NMR) spectroscopy. The MO6-24/O CPS polymer consists of four-sugar repeat subunits, each containing three residues of 2-acetamido-2,6-dideoxyhexopyranose in the $\alpha$-gluco configuration (trivially known as $\mathrm{N}$-acetyl quinovosamine (QuiNAc)) and one residue of 2-acetamido hexuronate in the $\alpha$-galactopyranose configuration ( $N$-acetyl-galactosamine uronic acid (GalNAcA)) [15]. Further structural details, including stereochemistry of sugar residues and position of glycosidic linkages, were provided by heteronuclear NMR spectroscopy and high-performance anion-exchange chromatography (HPAEC). In the same study, CPS with the identical composition was detected for the spontaneous translucent variant MO6-24/T, but not for an acapsular transposon mutant derivative [15]. Similar analysis of the CPS produced by another clinical isolate (BO62316) (Table 1) also revealed evidence of a four-sugar repeating subunits that included QuiNAc and GalNAcA residues but also included other sugars that were distinct from MO6-24/O CPS [16]. Analysis of the CPS from clinical isolate ATCC27562 showed further diversity with respect to composition, including the presence of $\mathrm{N}$-acetyl muramic acid, a well-known component of peptidoglycan, as well as evidence that the four-sugar repeat subunits for this strain are linked to serine via an amide bond [17].

Purified V. vulnificus CPS was poorly immunogenic in mice and rabbits [18]. However, use of CPS-protein conjugates did elicit improved immune responses such that the resulting antisera could be used to probe structural diversity of CPS. In agglutination assays, antibodies raised against CPS from strain C7184 (Table 1), for example, cross-reacted with only three other strains out of 32 tested [18], while antisera raised against strain MO6-24/O CPS cross-reacted with four out of 21 clinical isolates and none of 67 environmental strains examined [19]. In the latter study, NMR and HPAEC analyses of purified CPS from a subset of strains revealed most had distinct sugar composition and structure [19]. Such preliminary studies led to the development of a chemotyping method for V. vulnificus CPS, which involved HPAEC analysis coupled with electrochemical detection and computer automation. Analysis of CPS from $120 \mathrm{~V}$. vulnificus clinical and environmental isolates revealed that they could be divided into 94 'carbotypes', each differing in their carbohydrate composition [20]. No obvious correlation between carbotype and pathogenic potential of isolates was evident, though the possibility that some common feature (i.e., epitope) among clinical strains may exist could not be excluded [20]. 


\section{Further Insights Regarding CPS Functions That Contribute to Virulence}

As described earlier, studies conducted in the early to mid-1980s firmly established a correlation between virulence and CPS production in V. vulnificus. Moreover, specific virulence-related functions, such as antiphagocytic ability and resistance to the bacteriocidal action of human serum, appeared to be associated with CPS. Kreger et al. 1981 reported that a virulent strain of V. vulnificus was more resistant to phagocytosis by human polymorphonuclear (PMN) leukocytes than an attenuated strain, and their evidence suggested the possible involvement of an antiphagocytic antigen on the surface of the virulent isolate, which was later identified as an acidic polysaccharide [6,7]. Similarly, following exposure to opsonins present in NHS, highly virulent strains showed reduced uptake by human PMNs compared to less virulent isolates [21]. It was later shown that $V$. vulnificus also resisted phagocytosis by murine peritoneal macrophages in the absence of serum opsonins, a result which reinforced the notion that the surface of virulent cells (i.e., a molecule on the surface) had antiphagocytic properties [22].

Highly virulent $V$. vulnificus isolates also displayed resistance to the bacteriocidal action of NHS [21,23]. In assays designed to measure complement activation for killing, V. vulnificus activated significantly less complement than other pathogenic Vibrio spp. [23]. Multiple studies have since shown that opaque-colony-type variants of $V$. vulnificus are resistant to killing by NHS, while isogenic translucent variants are susceptible, which supports the hypothesis that CPS protects against serum killing $[9,24]$. It is possible that the negative charge of CPS confers resistance to the antibacterial components present in NHS [9,24]. Moreover, CPS may conceal immunogenic features on the surface of $V$. vulnificus cells that would otherwise facilitate the innate immune responses of the host, including phagocytosis by PMNs and macrophages and complement-mediated killing [2,3].

Septic shock typically results from excessive and systematic induction of inflammatory cytokines. For septicemia caused by $V$. vulnificus, such cytokines include tumor necrosis factor alpha (TNF- $\alpha$ ), interleukin 1 beta (IL-1 $\beta$ ), IL-6, and IL-8 [2,3]. A number of pathogen-associated molecules, including surface molecules, can interact with toll-like receptors (TLRs) present on host immune and non-immune cells to activate cytokine gene expression in a NF- $\mathrm{kB}$-dependent manner during infections [25]. Given the evidence that CPS is a major surface molecule on virulent $V$. vulnificus cells, a potential role for it in host cytokine activation was investigated. When exposed to either an encapsulated strain of $V$. vulnificus or purified CPS from that strain, the production of TNF- $\alpha$ was stimulated in vitro in human peripheral blood mononuclear cells or in vivo in mice [26]. Lee et al. (2010) then examined the effects on cytokine induction and related functions for human intestinal epithelial cells exposed to an encapsulated $V$. vulnificus clinical isolate versus an isogenic $\mathrm{CPS}$ mutant. The encapsulated strain induced significantly greater IL-8 production and NF- $\mathrm{KB}$ transcriptional activity than the mutant, and TLR-2 mRNA and protein levels were also greater following exposure to the encapsulated strain. Moreover, IL-8 production and NF- $\mathrm{kB}$ activity were also significantly induced following exposure to purified CPS, effects which could be blocked by the addition of anti-TLR2 antibodies. Thus, $V$. vulnificus CPS appears to induce inflammatory cytokine production via a TLR2/NF- $\mathrm{kB}$-dependent route [27].

An additional potential virulence-related function of the CPS of $V$. vulnificus is its apparent effect on production and spatial distribution of outer membrane vesicles (OMVs) [28]. As with other Gram-negative pathogens, OMVs may contribute to pathogenicity of $V$. vulnificus, a hypothesis supported by the finding that OMVs of this species induced death of epithelial cells by delivery of the cytolysin-hemolysin VvhA [29].

\section{CPS Genetics and Biosynthesis}

CPS biosynthesis and genetics in Escherichia coli has served as the model for characterization of capsules from other bacterial species. Based on CPS composition, as well as the underlying arrangement and complement of biosynthetic and transport genes, capsules of $E$. coli have been divided into four groups [30]. To date, the groups relevant for characterization of CPS production in V. vulnificus have been Groups 1 and 4. Capsules within Groups 1 and 4 are also interesting in that they are related to LPS O antigens [30]. 
Group 1 capsules of E. coli contain acidic sugar residues (e.g., uronic acids) as part of their repeat-unit structures, while Group 4 capsules are notable for the presence of acetomido sugars. For both capsular groups, individual repeat units are assembled on the lipid carrier undecaprenyl phosphate (und-P) with assembly occurring on the inner side of the inner membrane. Glycosyltransferase enzymes add the particular sugar residues that compose the repeat unit to the lipid carrier. In the case of Group 1 capsules, the WbaP protein, which is a member of the polyisoprenyl-phosphate hexose-1-phosphate transferase protein family, is the initiating glycosyltransferase, and it catalyzes the transfer of galactose-1-phosphate or glucose-1-phosphate to und-P, while WecA (polyisoprenyl-phosphate $\mathrm{N}$-acetylhexosamine-1-phosphate transferase family) performs the initial transfer of glucosamine-1-phosphate to und-P during Group 4 CPS synthesis. Following assembly, the completed und-P-linked repeat units for Group 1 and 4 capsules are translocated across the inner membrane by the Wzx flippase (polysaccharide transport (PST)-1 family; E.C. 7.5.2.6). The individual repeat units are then joined together on the periplasmic side of the inner membrane by the Wxy polymerase (no specified protein family), which transfers the growing polysaccharide from its lipid carrier to the newly translocated repeat unit [30].

CPS undergoes further polymerization and is relocated across the outer membrane of E. coli in a process that involves the Wza, Wzb, and Wzc proteins. Wza (outer membrane auxiliary (OMA) family) forms a multimeric pore through which the CPS traverses the outer membrane. Wzc is a tyrosine autokinase (and a member of the membrane periplasmic auxiliary (MPA)-1 protein family), which is anchored in the inner membrane but extends into the periplasm, and it is required for higher-level polymerization of CPS. Wzb (protein tyrosine phosphatase (PTP) family; E.C. 3.1.3.48) is the cognate phosphatase for Wzc. Both phosphorylation of Wzc and its dephosphorylation by Wzb are important for CPS synthesis [31]. Although Wza, Wzb, and Wzc proteins are required for both Group 1 and 4 CPS synthesis, a distinguishing feature is that the $w z a, w z b$, and $w z c$ genes are part of the Group 1 CPS biosynthetic and transport locus, which also includes $w z x$ and $w z y$, while in Group 4 strains, the $w z a$, $w z b$, and $w z c$ genes are encoded at a separate location from the main locus [30].

Sequencing of the genetic region flanking the sites of transposon insertions in acapsular mutants of various strains provided insights into the genetics of CPS synthesis in V. vulnificus. For strain MO6-24/O, the presence of both biosynthetic and transport genes, including $w z a, w z b$, and $w z c$, at a single locus and in an apparent single long operon configuration, coupled with the acidic composition of the MO6-24/O capsule, allowed for designation of a Group 1 capsule and CPS operon for this strain $[15,32,33]$.

Meanwhile, a partial genetic organization of the CPS locus in strain 1003(O) (Table 1) was reported $[12,14]$. Although the presence of a wecA homolog was suggestive of a Group 4 designation, a final group determination for strain $1003(\mathrm{O})$ was not possible from the available data [12].

Similar analysis of strain ATCC 27562 led to the identification of its CPS locus which had characteristics of Group 1 organization (i.e., linkage of $w z a, w z b$, and $w z c$ with CPS biosynthetic genes); however, the presence of a wecA glycosyltransferase gene at this locus, coupled with the CPS composition for this strain, supported a Group 4 designation $[17,34,35]$.

The findings of linkage between CPS transport and biosynthetic genes even for the Group 4 strain ATCC 27562 raise the possibility that such gene organization may be common in this species. As an initial test of this notion, we determined the position of the $w z a, w z b$, and $w z c$ genes relative to the CPS biosynthetic locus in $21 \mathrm{~V}$. vulnificus strains whose complete genome sequences were available in the NCBI database. We found evidence for such linkage in 14 of those strains, while five strains showed no linkage between transport and biosynthetic genes, and two could not be determined due to limited sequence identity of the genes involved. Among the 14 strains that showed linkage, the majority possessed a $w b p A$ homolog, though there were also multiple strains, besides ATCC 27562, that contained wecA (Mukerji and Pettis, unpublished results).

Interestingly, the CPS locus of strain ATCC 27562 was also found to be largely conserved and syntenous with one in the marine bacterium Shewanella putrefaciens strain 200 [34]. The average GC content for both loci was lower than that of either host genome, and a number of the encoded proteins 
showed highest similarity to proteins of Gram-positive and archaeal origins. These findings led to the authors' proposed hypothesis that these CPS loci were acquired by their V. vulnificus and S. putrefaciens host strains via horizontal gene transfer (HGT) events from an unrelated microbe [34].

In a follow-up study, the potential for HGT acquisition of CPS loci by V. vulnificus was explored further. It was found that $V$. vulnificus strains induced to a state of competence during transformation following exposure to chitin were able to incorporate into their genome exogenous DNA, including CPS locus sequences tagged with a selectable marker. In some cases, full or partial CPS locus sequences were incorporated, which resulted in a change in CPS carbotype [36]. It is possible that the notable CPS carbotype diversity in V. vulnificus [20] has been the result, at least in part, of HGT acquisition of CPS loci, which can result in new combinations of biosynthetic genes and therefore new carbotypes of CPS [36].

\section{CPS Phase Variation}

Both opaque and translucent colony types of $V$. vulnificus strains were often readily observed [8,9,37], thus raising the possibility that phase variation between these two morphotypes was occurring. Rates of switching from opaque to translucent colonial variants ranged from $10^{-4}$ to $10^{-5}[9,11,38]$; in some cases, phenotypic switching from translucent back to opaque at rates on the order of $10^{-4}$ was also observed $[9,11]$. Such frequencies, which were orders of magnitude greater than expected if spontaneous mutations were responsible, supported the existence of a reversible CPS phase variation mechanism(s).

Chatzidaki-Livanis et al. (2006) then studied the process of phase variation in more detail at both the phenotypic and genotypic levels for V. vulnificus strains that contained a Group 1 CPS operon. They found evidence for two distinct types of translucent isolates derived from an opaque parent. One translucent type retained the ability to switch back to the opaque form, while the other type showed no evidence of switching and thus appeared to be phase locked. In electron micrographs of negatively stained cells, translucent isolates capable of phase switching showed reduced amounts of CPS on the cell surface as compared to the opaque parent, while phase-locked translucent isolates appeared to be acapsular. At the genetic level, PCR analysis revealed that phase-locked translucent isolates had typically undergone deletion of the $w z b$ gene along with some flanking sequences within the Group 1 CPS operon (e.g., TR2 isolates). Meanwhile, translucent isolates that retained the ability for reversible phase variation (TR1) possessed no mutations at all within their Group 1 operons [32].

In the same study, examination of Group 1 operon sequences revealed a probable mechanism for the creation of phase-locked translucent variants and uncovered allelic differences at this locus. Opaque strains containing allele 1 (e.g., MO6-24/O) were distinguished by having multiple copies of the same octamer sequence (ACAGGACC) repeated directly and sequentially both upstream and downstream of $w z b$. Opaque strains containing allele 2 also had multiple direct repeats present within their Group 1 transport regions, including on either side of $w z b$. However, the sequence of these repeats was more variable (A/CCTAGG/AAA/C) and copies were interspersed with other unrelated sequences. As shown in Figure 2, a potential mechanism for the generation of phase-locked translucent variants would then consist of homologous recombination involving repeat sequences flanking $w z b$, which would result in deletion of the intervening sequence [32].

The intermediate (Int) variant reported for certain strains of $V$. vulnificus appears to be analogous to the TR1 isolate, in that both contained an intact $w z b$ gene and could switch back to opaque [3,32,39]. RT-PCR analysis suggested the possibility of transient down-regulation of $w z b$ expression in newly formed Int colonies [39]. The potential for expression differences in Group 1 CPS operon genes in opaque versus TR1 (Int) derivatives remains an interesting possibility to be explored. 


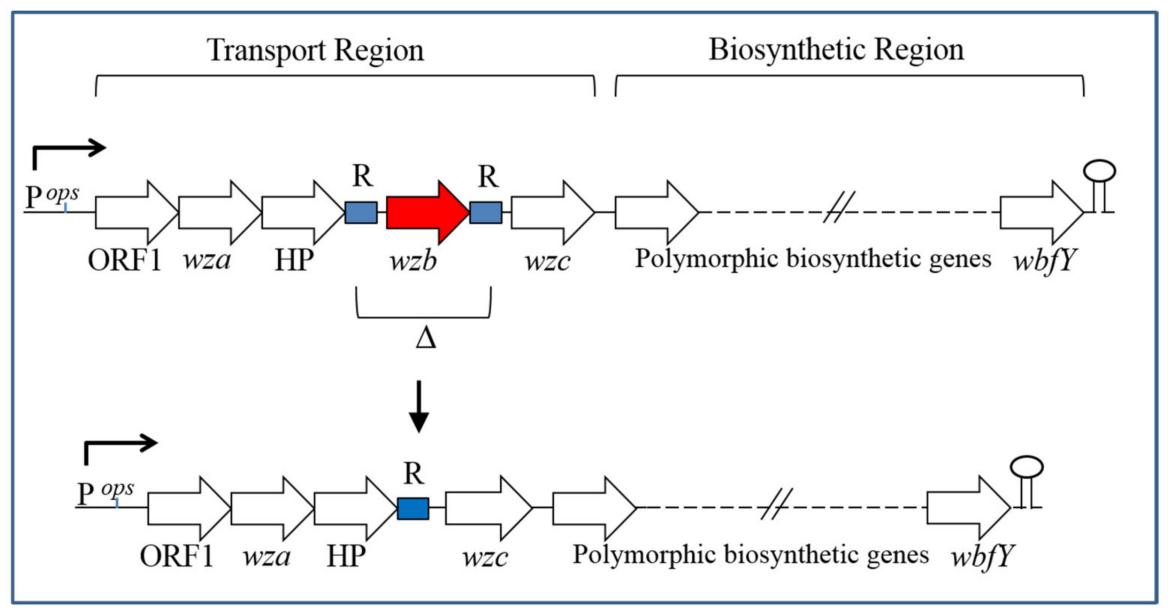

Figure 2. Model for generation of phase-locked translucent variants based on Chatzidaki-Livanis et al. (2006). The upper portion of the figure depicts the general organization of the Group 1 CPS operon in $V$. vulnificus, including the positions of the essential $w z b$ phosphatase gene and repeat sequence (R) regions flanking that gene. For strains containing allele 1 of this operon, each $\mathrm{R}$ region contains multiple copies of the octamer (ACAGGACC). Allele 2 strains have an additional R region (not shown) located between $w z a$ and HP (hypothetical protein gene), and each $\mathrm{R}$ region contains multiple copies of (A/CCTAGG/AAA/C) [32]. Starting with an opaque variant, homologous recombination between $\mathrm{R}$ regions with deletion of intervening sequences, including $w z b$, would generate a phase-locked translucent variant (i.e., as depicted in the lower portion of the figure) [32]. The relative positions of the operon promoter $(\mathrm{P})$, ops element, and Rho-independent transcription terminator hairpin structure are indicated.

A number of environmental factors affecting CPS phase variation in $V$. vulnificus have now been identified. Significantly greater switching from opaque to translucent was seen during static growth as compared to growth under aeration for most strains analyzed [40]. Greater opaque-to-translucent switching was also seen at elevated (e.g., $\left.37^{\circ} \mathrm{C}\right)$ versus lower $\left(23^{\circ} \mathrm{C}\right)$ temperatures [40]. These authors also compared phase switching from opaque to translucent in response to temperature for $C$ type (i.e., predominantly clinical isolates) versus $\mathrm{E}$ type (predominantly environmental isolates) of $V$. vulnificus strains, which had previously been subdivided into those types by using a randomly amplified polymorphic DNA-PCR method [41]. Interestingly, C-type strains were significantly less prone than $\mathrm{E}$ type strains to switch to the translucent form at all temperatures examined including those relevant to human infection [40].

In an interesting twist on the phase variation phenomenon, it was found that opaque V. vulnificus strains appeared translucent when grown under anaerobic conditions [42]. However, such strains regained opacity when exposed to an aerobic environment. In line with these phenotypic results, it was found that expression of the $w z a, w z b$, and $w z c$ genes were all significantly downregulated under anaerobic conditions. These results raise the possibility that CPS production may be downregulated during earlier anaerobic stages of human infection, such as colonization of the intestinal tract [42].

Calcium $\left(\mathrm{Ca}^{2+}\right)$ was found to significantly enhance colonial phase variation of $V$. vulnificus. Some opaque strains switched predominantly to translucent in the presence of elevated calcium while other strains switched mostly to a biofilm-proficient rugose form [43]. Increased manganese $\left(\mathrm{Mn}^{2+}\right)$ concentrations also significantly enhanced switching of opaque strains to translucent or rugose phenotypes [44]. PCR analysis of translucent isolates obtained following growth in elevated $\mathrm{Ca}^{2+}$ or $\mathrm{Mn}^{2+}$ revealed the presence of both $w z b+$ translucent variants, as well as many that had undergone deletions of $w z b$. Thus, elevated $\mathrm{Ca}^{2+}$ or $\mathrm{Mn}^{2+}$ concentrations, which the bacterium may encounter in its natural estuarine environment, likely up-regulate existing phase variation mechanisms [43,44].

In its natural marine habitat, V. vulnificus becomes concentrated in filter feeders such as oysters. Using an oyster model of infection that was reduced for indigenous $V$. vulnificus populations, Srivastava et al. (2009) studied the ability of various $V$. vulnificus mutants and phase variants to 
colonize and persist in oysters. Reminiscent to what was seen previously in mice [11], it was found that following infection of oysters with a T1 (TR1) translucent isolate, the strain was subsequently recovered in the opaque form at a substantial frequency (i.e., $72 \%$ of the colony-forming units recovered from the oysters were opaque while only $28 \%$ remained translucent) [45]. The predominance of the opaque form over the translucent one in oysters was supported by co-infection experiments [45], as well as by previous observations that translucent $V$. vulnificus isolates appear to be more susceptible than opaque to phagocytosis by oyster hemocytes [46].

Some regulatory systems also appear to affect phase variation in V. vulnificus. GacS/GacA is a two-component signal transduction system that regulates a variety of functions in Gram-negative bacteria, including virulence factor production [47]. While a gac $A$ knockout strain of $V$. vulnificus retained colony opacity, its ability to undergo phase switching to translucent was significantly reduced [48]. Among the transcripts significantly down-regulated in the gacA mutant was rpoS [48], which encodes the sigma factor $\left(\sigma^{S}\right)$ that is critical for stationary phase gene expression. Phase variation in V. vulnificus has been purported to be a response to stationary growth [32]; thus, the results for $g a c A$ and $r p o S$ may shed light on one regulatory mechanism controlling the appearance of new phase variants in this species.

In a separate study, insertion into or deletion of the rseB gene, produced mutants that appeared less opaque than the parental strain and, upon passaging, yielded mixed populations of opaque and translucent colonies at a high frequency [49]. The RseB protein is a periplasmic negative regulator of $\sigma^{\mathrm{E}}$, which controls expression of genes critical for maintaining cell envelope integrity during stress (i.e., the extracytoplasmic stress response) [50]. A strain overexpressing $\sigma^{\mathrm{E}}$ did not recapitulate the colonial phenotype and phase-switching characteristics of $r s e B$ mutants [49]. Nevertheless, the results suggested a potential role for $r s e B$ in regulating phase variation in $V$. vulnificus, although the extent to which CPS expression specifically was affected in these mutants was not determined [49].

\section{Other Regulation of CPS}

While not contributing to phase variation per se, other environmental and genetics factors have also been identified that affect CPS expression in V. vulnificus. Using a fluorescence-based flow cytometry method specific for CPS, Wright et al. (1999) determined that encapsulated strains showed maximal CPS expression during logarithmic growth as opposed to the stationary phase. Moreover, opaque cells produced significantly more CPS when grown at $30^{\circ} \mathrm{C}$ rather than $37^{\circ} \mathrm{C}$ [51].

Biofilm production in V. vulnificus involves an exopolysaccharide that is distinct from CPS and is encoded by its own exopolysaccharide gene cluster [52,53]. Joseph and Wright (2004) compared the ability of isogenic opaque and translucent variants to form biofilms and found that translucent isolates produced significantly more biofilm than opaque. The authors concluded that CPS inhibits biofilm formation in V. vulnificus [54]. Lee et al. (2013) then showed that exogenous addition of purified CPS to cultures of a translucent variant greatly reduced biofilm formation, while addition of a CPS-degrading enzyme to the parental opaque strain significantly increased its ability to form biofilms. They next demonstrated that CPS inhibits biofilm formation in a quorum sensing-dependent manner. A mutant defective for the quorum sensing master regulator SmcR had a translucent phenotype on agar plates, and transcription of the Group 1 CPS operon was reduced tenfold in the $s m c R$ mutant compared to the opaque wild type parent. A SmcR binding sequence in the promoter region for this operon was demonstrated by using DNaseI footprinting assays involving recombinant SmcR protein. Overall, the authors suggested that CPS may play an important role in regulating the size of mature biofilms by limiting their continual growth [55].

In a follow-up study, CPS production and CPS Group 1 operon gene expression were found to be significantly reduced in cells subjected to heat shock at $42{ }^{\circ} \mathrm{C}$. Basal level transcription of the CPS operon under these conditions was shown to be the result of enhanced degradation of SmcR by proteases ClpPA and Lon [56]. 
Lastly, the production of a variety of surface molecules in Gram-negative species has been shown to be regulated by the antiterminator $\mathrm{RfaH}$ protein, which functions to prevent premature transcriptional termination at Rho-dependent sites within the cognate operons for these molecules. $\mathrm{RfaH}$ is recruited to the transcription elongation complex by binding a small conserved operon polarity sequence (ops), which is typically located upstream of the first gene within operons [57,58]. Given that an ops element was previously described [33] within the Group 1 CPS operon of V. vulnificus (Figure 2), an effort was undertaken to evaluate the role of $\mathrm{RfaH}$ in CPS production in this species. It was found that knockout of the rfaH gene in an opaque parental strain produced a translucent derivative that appeared phase locked. Moreover, distal, but not proximal, gene expression within the CPS Group 1 operon was significantly reduced in the $r f a H$ mutant, and it was highly sensitive to killing by NHS. Thus, CPS production and serum survival in V. vulnificus were found to be dependent on RfaH antitermination control [59].

\section{Concluding Remarks}

The CPS of $V$. vulnificus plays pivotal roles in circumventing the innate immune response during infection of the human host, and it appears to also stimulate a portion of the inflammatory cytokine response to this pathogen. Substantial CPS carbotype diversity among V. vulnificus strains is underscored by considerable genetic diversity, which may be the result of HGT events that lead to new combinations of CPS biosynthetic genes. As summarized in Figure 3, CPS production is controlled by numerous environmental, genetic, and regulatory factors, including those that affect phase variation between virulent encapsulated opaque variants and attenuated translucent variants, which possess little or no CPS on their cell surface. Further study of CPS production and its regulation in $V$. vulnificus should continue to provide important insights that may ultimately lead to improved disease management of this deadly pathogen.

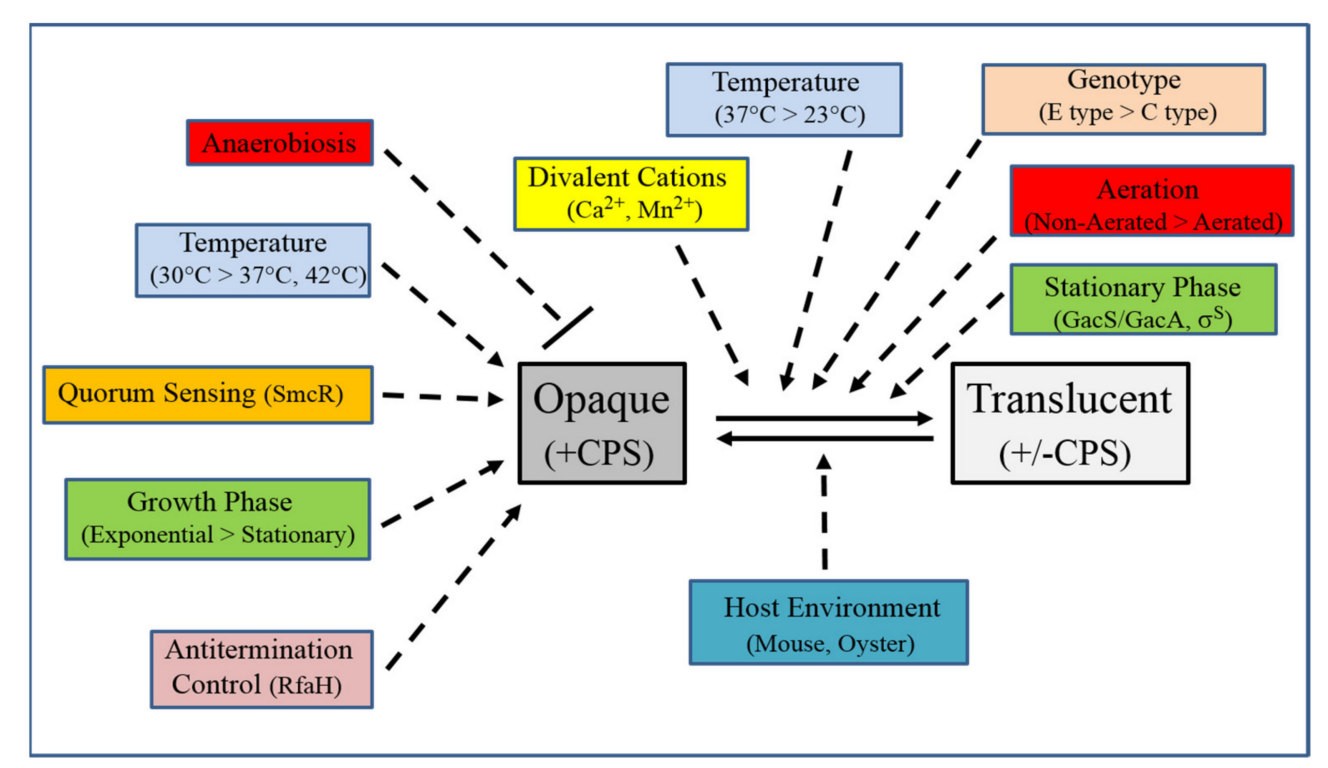

Figure 3. Summary of known environmental, genetic, and regulatory factors that affect CPS production in V. vulnificus. CPS production in opaque variants is negatively affected (depicted by dashed line with bar) by anaerobiosis [42], but positively affected (dashed arrows) by lower growth temperature [51,56], quorum sensing as mediated by SmcR [55], exponential growth [51], and antitermination control via $\mathrm{RfaH}$ [59]. Phase switching from opaque to translucent (solid arrow) is positively affected by divalent cations $\mathrm{Ca}^{2+}$ and $\mathrm{Mn}^{2+}[43,44]$, higher growth temperature [40], E-type genotypes [40], non-aerated growth [40], and stationary phase growth, with the latter effect potentially being mediated by GacS/GacA and $\sigma^{S}$ [48]. Host environments such as mouse [11] and oyster [45] promote phase variation from translucent to opaque, but to what extent, if any, the rate of phase switching is affected by these environments remains to be determined. See text for more details regarding these various factors. 
Funding: Research described from the authors' lab was supported in part by the Louisiana Sea Grant College Program with funds (to G.S.P.) from the National Oceanic and Atmospheric Administration Office of Sea Grant, Department of Commerce, under grant no. NA06OAR4170022, project no. R/PMO-21 and Louisiana State University. Statements, findings, conclusions, and recommendations are those of the authors and do not necessarily reflect the views of either the Louisiana Sea Grant College Program or the U.S. Department of Commerce.

Acknowledgments: Light microscopy was performed at the Shared Instrumentation Facility (SIF) at Louisiana State University. We thank past members of the lab, including Brenda Grau, Katherine Garrison-Schilling, Zelam Kaluskar, Shana Garrett, and Bliss Lambert, for their contributions towards elucidating aspects of phase variation and polysaccharide production in $V$. vulnificus.

Conflicts of Interest: The authors declare no conflict of interest. The funders had no role in the design or writing of the manuscript or in the decision to publish it.

\section{Abbreviations}

$\begin{array}{ll}\text { CPS } & \text { Capsular polysaccharide } \\ \text { LPS } & \text { Lipopolysaccharide } \\ \text { NHS } & \text { Normal human serum } \\ \text { NMR } & \text { Nuclear magnetic resonance } \\ \text { HPAEC } & \text { High performance anion-exchange chromatography } \\ \text { TNF- } \alpha & \text { Tumor necrosis factor alpha } \\ \text { IL } & \text { Interleukin } \\ \text { Und-P } & \text { Undecaprenyl phosphate }\end{array}$

\section{References}

1. Oliver, J.D. Vibrio vulnificus: Death on the half shell. A personal journey with the pathogen and its ecology. Microb. Ecol. 2013, 65, 793-799. [PubMed]

2. Horseman, M.A.; Surani, S. A comprehensive review of Vibrio vulnificus: An important cause of severe sepsis and skin and soft-tissue infection. Int. J. Infect. Dis. 2011, 15, e157-e166. [CrossRef] [PubMed]

3. Jones, M.K.; Oliver, J.D. Vibrio vulnificus: Disease and pathogenesis. Infect. Immun. 2009, 77, 1723-1733.

4. Oliver, J.D. Wound infections caused by Vibrio vulnificus and other marine bacteria. Epidemiol. Infect. 2005, 133, 383-391.

5. Gulig, P.A.; Bourdage, K.L.; Starks, A.M. Molecular Pathogenesis of Vibrio vulnificus. J. Microbiol. 2005, 43, 118-131.

6. Kreger, A.; DeChatelet, L.; Shirley, P. Interaction of Vibrio vulnificus with human polymorphonuclear leukocytes: Association of virulence with resistance to phagocytosis. J. Infect. Dis. 1981, 144, $244-248$. [CrossRef]

7. Kreger, A.S.; Gray, L.D.; Testa, J. Protection of mice against Vibrio vulnificus disease by vaccination with surface antigen preparations and anti-surface antigen antisera. Infect. Immun. 1984, 45, 537-543.

8. Amako, K.; Okada, K.; Miake, S. Evidence for the presence of a capsule in Vibrio vulnificus. J. Gen. Microbiol. 1984, 130, 2741-2743. [CrossRef]

9. Yoshida, S.; Ogawa, M.; Mizuguchi, Y. Relation of capsular materials and colony opacity to virulence of Vibrio vulnificus. Infect. Immun. 1985, 47, 446-451. [CrossRef]

10. Simpson, L.M.; White, V.K.; Zane, S.F.; Oliver, J.D. Correlation between virulence and colony morphology in Vibrio vulnificus. Infect. Immun. 1987, 55, 269-272. [CrossRef]

11. Wright, A.C.; Simpson, L.M.; Oliver, J.D.; Morris, J.G., Jr. Phenotypic evaluation of acapsular transposon mutants of Vibrio vulnificus. Infect. Immun. 1990, 58, 1769-1773. [CrossRef] [PubMed]

12. Smith, A.B.; Siebeling, R.J. Identification of genetic loci required for capsular expression in Vibrio vulnificus. Infect. Immun. 2003, 71, 1091-1097. [CrossRef] [PubMed]

13. Yamazaki, K.; Kashimoto, T.; Morita, M.; Kado, T.; Matsuda, K.; Yamasaki, M.; Ueno, S. Identification of in vivo Essential Genes of Vibrio vulnificus for Establishment of Wound Infection by Signature-Tagged Mutagenesis. Front. Microbiol. 2019, 10, 123. [CrossRef] [PubMed]

14. Zuppardo, A.B.; Siebeling, R.J. An epimerase gene essential for capsule synthesis in Vibrio vulnificus. Infect. Immun. 1998, 66, 2601-2606. [CrossRef] [PubMed] 
15. Reddy, G.P.; Hayat, U.; Abeygunawardana, C.; Fox, C.; Wright, A.C.; Maneval, D.R., Jr.; Bush, C.A.; Morris, J.G., Jr. Purification and determination of the structure of capsular polysaccharide of Vibrio vulnificus MO6-24. J. Bacteriol. 1992, 174, 2620-2630. [CrossRef] [PubMed]

16. Reddy, G.P.; Hayat, U.; Bush, C.A.; Morris, J.G., Jr. Capsular polysaccharide structure of a clinical isolate of Vibrio vulnificus strain BO62316 determined by heteronuclear NMR spectroscopy and high-performance anion-exchange chromatography. Anal. Biochem. 1993, 214, 106-115. [CrossRef]

17. Gunawardena, S.; Reddy, G.P.; Wang, Y.; Kolli, V.S.; Orlando, R.; Morris, J.G.; Bush, C.A. Structure of a muramic acid containing capsular polysaccharide from the pathogenic strain of Vibrio vulnificus ATCC 27562. Carbohydr. Res. 1998, 309, 65-76. [CrossRef]

18. Simonson, J.G.; Siebeling, R.J. Immunogenicity of Vibrio vulnificus capsular polysaccharides and polysaccharide-protein conjugates. Infect. Immun. 1993, 61, 2053-2058. [CrossRef]

19. Hayat, U.; Reddy, G.P.; Bush, C.A.; Johnson, J.A.; Wright, A.C.; Morris, J.G., Jr. Capsular types of Vibrio vulnificus: An analysis of strains from clinical and environmental sources. J. Infect. Dis. 1993, 168, 758-762. [CrossRef]

20. Bush, C.A.; Patel, P.; Gunawardena, S.; Powell, J.; Joseph, A.; Johnson, J.A.; Morris, J.G. Classification of Vibrio vulnificus strains by the carbohydrate composition of their capsular polysaccharides. Anal. Biochem. 1997, 250, 186-195. [CrossRef]

21. Johnson, D.E.; Calia, F.M.; Musher, D.M.; Goree, A. Resistance of Vibrio vulnificus to serum bactericidal and opsonizing factors: Relation to virulence in suckling mice and humans. J. Infect. Dis. 1984, 150, 413-418. [CrossRef] [PubMed]

22. Tamplin, M.L.; Specter, S.; Rodrick, G.E.; Friedman, H. Vibrio vulnificus resists phagocytosis in the absence of serum opsonins. Infect. Immun. 1985, 49, 715-718. [CrossRef] [PubMed]

23. Tamplin, M.L.; Specter, S.; Rodrick, G.E.; Friedman, H. Differential complement activation and susceptibility to human serum bactericidal action by Vibrio species. Infect. Immun. 1983, 42, 1187-1190. [CrossRef]

24. Williams, T.C.; Ayrapetyan, M.; Ryan, H.; Oliver, J.D. Serum survival of Vibrio vulnificus: Role of genotype, capsule, complement, clinical origin, and in situ incubation. Pathogens 2014, 3, 822-832. [CrossRef]

25. Kawasaki, T.; Kawai, T. Toll-like receptor signaling pathways. Front. Immunol. 2014, 5, 461. [CrossRef] [PubMed]

26. Powell, J.L.; Wright, A.C.; Wasserman, S.S.; Hone, D.M.; Morris, J.G., Jr. Release of tumor necrosis factor alpha in response to Vibrio vulnificus capsular polysaccharide in in vivo and in vitro models. Infect. Immun. 1997, 65, 3713-3718. [CrossRef] [PubMed]

27. Lee, B.C.; Kim, M.S.; Choi, S.H.; Kim, T.S. Involvement of capsular polysaccharide via a TLR2/NF-kappaB pathway in Vibrio vulnificus-induced IL-8 secretion of human intestinal epithelial cells. Int. J. Mol. Med. 2010, 25, 581-591. [PubMed]

28. Hampton, C.M.; Guerrero-Ferreira, R.C.; Storms, R.E.; Taylor, J.V.; Yi, H.; Gulig, P.A.; Wright, E.R. The Opportunistic Pathogen Vibrio vulnificus Produces Outer Membrane Vesicles in a Spatially Distinct Manner Related to Capsular Polysaccharide. Front. Microbiol. 2017, 8, 2177. [CrossRef]

29. Kim, Y.R.; Kim, B.U.; Kim, S.Y.; Kim, C.M.; Na, H.S.; Koh, J.T.; Choy, H.E.; Rhee, J.H.; Lee, S.E. Outer membrane vesicles of Vibrio vulnificus deliver cytolysin-hemolysin VvhA into epithelial cells to induce cytotoxicity. Biochem. Biophys. Res. Commun. 2010, 399, 607-612. [CrossRef]

30. Whitfield, C. Biosynthesis and assembly of capsular polysaccharides in Escherichia coli. Annu. Rev. Biochem. 2006, 75, 39-68. [CrossRef]

31. Whitfield, C.; Paiment, A. Biosynthesis and assembly of Group 1 capsular polysaccharides in Escherichia coli and related extracellular polysaccharides in other bacteria. Carbohydr. Res. 2003, 338, 2491-2502. [CrossRef] [PubMed]

32. Chatzidaki-Livanis, M.; Jones, M.K.; Wright, A.C. Genetic variation in the Vibrio vulnificus group 1 capsular polysaccharide operon. J. Bacteriol. 2006, 188, 1987-1998. [CrossRef] [PubMed]

33. Wright, A.C.; Powell, J.L.; Kaper, J.B.; Morris, J.G., Jr. Identification of a group 1-like capsular polysaccharide operon for Vibrio vulnificus. Infect. Immun. 2001, 69, 6893-6901. [CrossRef] [PubMed]

34. Nakhamchik, A.; Wilde, C.; Chong, H.; Rowe-Magnus, D.A. Evidence for the horizontal transfer of an unusual capsular polysaccharide biosynthesis locus in marine bacteria. Infect. Immun. 2010, 78, 5214-5222. [CrossRef] 
35. Nakhamchik, A.; Wilde, C.; Rowe-Magnus, D.A. Identification of a Wzy polymerase required for group IV capsular polysaccharide and lipopolysaccharide biosynthesis in Vibrio vulnificus. Infect. Immun. 2007, 75, 5550-5558. [CrossRef]

36. Neiman, J.; Guo, Y.; Rowe-Magnus, D.A. Chitin-induced carbotype conversion in Vibrio vulnificus. Infec Immun. 2011, 79, 3195-3203. [CrossRef]

37. Moreno, M.L.; Landgraf, M. Virulence factors and pathogenicity of Vibrio vulnificus strains isolated from seafood. J. Appl. Microbiol. 1998, 84, 747-751. [CrossRef]

38. Kim, C.M.; Jeong, K.C.; Rhee, J.H.; Choi, S.H. Thermal-death times of opaque and translucent morphotypes of Vibrio vulnificus. Appl. Environ. Microbiol. 1997, 63, 3308-3310. [CrossRef]

39. Rosche, T.M.; Smith, B.; Oliver, J.D. Evidence for an intermediate colony morphology of Vibrio vulnificus. Appl. Environ. Microbiol. 2006, 72, 4356-4359. [CrossRef]

40. Hilton, T.; Rosche, T.; Froelich, B.; Smith, B.; Oliver, J. Capsular polysaccharide phase variation in Vibrio vulnificus. Appl. Environ. Microbiol. 2006, 72, 6986-6993. [CrossRef]

41. Rosche, T.M.; Yano, Y.; Oliver, J.D. A rapid and simple PCR analysis indicates there are two subgroups of Vibrio vulnificus which correlate with clinical or environmental isolation. Microbiol. Immunol. 2005, 49, 381-389. [CrossRef] [PubMed]

42. Phippen, B.L.; Oliver, J.D. Role of anaerobiosis in capsule production and biofilm formation in Vibrio vulnificus. Infect. Immun. 2015, 83, 551-559. [CrossRef] [PubMed]

43. Garrison-Schilling, K.L.; Grau, B.L.; McCarter, K.S.; Olivier, B.J.; Comeaux, N.E.; Pettis, G.S. Calcium promotes exopolysaccharide phase variation and biofilm formation of the resulting phase variants in the human pathogen Vibrio vulnificus. Environ. Microbiol. 2011, 13, 643-654. [CrossRef] [PubMed]

44. Kaluskar, Z.M.; Garrison-Schilling, K.L.; McCarter, K.S.; Lambert, B.; Simar, S.R.; Pettis, G.S. Manganese is an additional cation that enhances colonial phase variation of Vibrio vulnificus. Environ. Microbiol. Rep. 2015, 7, 789-794. [CrossRef]

45. Srivastava, M.; Tucker, M.S.; Gulig, P.A.; Wright, A.C. Phase variation, capsular polysaccharide, pilus and flagella contribute to uptake of Vibrio vulnificus by the Eastern oyster (Crassostrea virginica). Environ. Microbiol. 2009, 11, 1934-1944. [CrossRef]

46. Harris-Young, L.; Tamplin, M.L.; Fisher, W.S.; Mason, J.W. Effects of Physicochemical Factors and Bacterial Colony Morphotype on Association of Vibrio vulnificus with Hemocytes of Crassostrea virginica. Appl. Environ. Microbiol. 1993, 59, 1012-1017. [CrossRef]

47. Heeb, S.; Haas, D. Regulatory roles of the GacS/GacA two-component system in plant-associated and other gram-negative bacteria. Mol. Plant Microbe Interact. 2001, 14, 1351-1363. [CrossRef]

48. Gauthier, J.D.; Jones, M.K.; Thiaville, P.; Joseph, J.L.; Swain, R.A.; Krediet, C.J.; Gulig, P.A.; Teplitski, M.; Wright, A.C. Role of GacA in virulence of Vibrio vulnificus. Microbiology 2010, 156, 3722-3733. [CrossRef]

49. Brown, R.N.; Gulig, P.A. Roles of RseB, sigmaE, and DegP in virulence and phase variation of colony morphotype of Vibrio vulnificus. Infect. Immun. 2009, 77, 3768-3781. [CrossRef]

50. DeAngelis, C.M.; Saul-McBeth, J.; Matson, J.S. Vibrio responses to extracytoplasmic stress. Environ. Microbiol. Rep. 2018, 10, 511-521. [CrossRef]

51. Wright, A.C.; Powell, J.L.; Tanner, M.K.; Ensor, L.A.; Karpas, A.B.; Morris, J.G., Jr.; Sztein, M.B. Differential expression of Vibrio vulnificus capsular polysaccharide. Infect. Immun. 1999, 67, 2250-2257. [CrossRef] [PubMed]

52. Garrison-Schilling, K.L.; Kaluskar, Z.M.; Lambert, B.; Pettis, G.S. Genetic analysis and prevalence studies of the brp exopolysaccharide locus of Vibrio vulnificus. PLoS ONE 2014, 9, e100890. [CrossRef] [PubMed]

53. Guo, Y.; Rowe-Magnus, D.A. Identification of a c-di-GMP-regulated polysaccharide locus governing stress resistance and biofilm and rugose colony formation in Vibrio vulnificus. Infect. Immun. 2010, 78, 1390-1402. [CrossRef] [PubMed]

54. Joseph, L.A.; Wright, A.C. Expression of Vibrio vulnificus capsular polysaccharide inhibits biofilm formation. J. Bacteriol. 2004, 186, 889-893. [CrossRef]

55. Lee, K.J.; Kim, J.A.; Hwang, W.; Park, S.J.; Lee, K.H. Role of capsular polysaccharide (CPS) in biofilm formation and regulation of CPS production by quorum-sensing in Vibrio vulnificus. Mol. Microbiol. 2013, 90, 841-857. [CrossRef]

56. Lee, K.J.; Jung, Y.C.; Park, S.J.; Lee, K.H. Role of Heat Shock Proteases in Quorum-Sensing-Mediated Regulation of Biofilm Formation by Vibrio Species. MBio 2018, 9. [CrossRef] 
57. Bailey, M.J.; Hughes, C.; Koronakis, V. RfaH and the ops element, components of a novel system controlling bacterial transcription elongation. Mol. Microbiol. 1997, 26, 845-851. [CrossRef]

58. Yakhnin, A.V.; Babitzke, P. NusG/Spt5: Are there common functions of this ubiquitous transcription elongation factor? Curr. Opin. Microbiol. 2014, 18, 68-71. [CrossRef]

59. Garrett, S.B.; Garrison-Schilling, K.L.; Cooke, J.T.; Pettis, G.S. Capsular polysaccharide production and serum survival of Vibrio vulnificus are dependent on antitermination control by RfaH. FEBS Lett. 2016, 590, 4564-4572. [CrossRef]

(C) 2020 by the authors. Licensee MDPI, Basel, Switzerland. This article is an open access article distributed under the terms and conditions of the Creative Commons Attribution (CC BY) license (http://creativecommons.org/licenses/by/4.0/). 